\title{
De la olla común \\ a la acción colectiva, las mujeres “Yela” en Talca, 1980-1995*
}

\author{
Hillary Hiner
}

Universidad Diego Portales, Santiago, Chile. Email: hillary.hiner@udp.cl

\begin{abstract}
Resumen: En 1986 se formó en Talca la Casa Yela, una de las primeras organizaciones de mujeres que luchaba en contra de la violencia doméstica y sexual. Compuesta principalmente de mujeres populares, esta pequeña ONG logró establecer su propia casa de acogida en 1995. Lo que nos interesa explorar en este artículo es la manera en que se fue articulando la Casa Yela, a nivel endógeno y exógeno, en términos de su configuración como grupo y su inserción dentro de una red de otras organizaciones. De particular interés para la literatura especializada sobre movimientos sociales y mujeres populares, nuestro estudio propone que la Casa Yela enfrentó principalmente dos desafíos constantes durante su trayectoria -una falta crónica de recursos y una relación fluctuante con el Estado. Exploraremos las maneras en que la Casa Yela manejó este panorama, y utilizaremos este caso de estudio para desdibujar algunas ideas sobre la relación entre las organizaciones de base y el Estado durante el período de estudio.

Palabras clave: mujeres populares, violencia contra la mujer, movimientos sociales, Estado
\end{abstract}

\section{From the "common pot" to collective action, the "Yela" women in Talca, 1980-1995}

\begin{abstract}
In 1986, one of the first organizations was formed in Talca to combat domestic and sexual violence was created, Casa Yela. Conformed primarily by popular-sector women, this small NGO was able to establish its own battered women's shelter in 1995. What most interests us in this article is the way in which Casa Yela was formed, on an endogenous and exogenous level, in terms of its configuration as a group and its insertion within a web of other organizations. Of particular interest to the specialized literature on social movements and popularsector women, our study proposes that Casa Yela rose up against two main challenges during its development -a chronic lack of resources and a fluctuating relationship with the state. We will explore the ways in which Casa Yela navigated this situation and we will use this case study to sketch some ideas about the relationship between grassroots organizations and the state during the period of study.

Key words: popular-sector women, violence against women, social movements, State
\end{abstract}

\section{Do fundo comum para a ação coletiva, as mulheres "Yela" em Talca, 1980-1995}

Resumo: Em 1986 foi formado em Talca Casa Yela, uma das primeras 
organizações de mulheres da luta contra a violência doméstica e sexual. Composta principalmente por mulheres pobres, esta pequena ONG criou o seu próprio abrigo, em 1995. O que queremos explorar neste artigo é a forma como foi-se articulando a Casa Yela, ao nível endógeno e exógeno, em termos de seus próprios como um grupo e sua integração dentro de uma rede de outras organizações. De particular interesse para a literatura sobre movimentos sociais e mulheres popular, nosso estudo sugere que a Casa Yela enfrentou dois grandes desafios constantes ao longo de sua carreira - uma crônica falta de recursos e uma relação de flutuação com o estado. Explorar de que maneira a Casa Yela Casa tratou esta situação, e usará-se este estudo de caso para esbater algumas idéias sobre a relação organizações populares/Estado durante o período do estudo.

Palavras-chave: mulheres populares, violência contra as mulheres, movimentos sociais, Estado

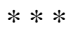

En este artículo estudiaremos la formación de un pequeño grupo de mujeres populares que se unieron en el sector norte de Talca durante los años 80. Este grupo, que se conocería más adelante como la “Casa Yela”, tiene sus raíces en una serie de corrientes movimentistas prominentes durante la dictadura -las comunidades eclesiales de base de la Iglesia, los comedores populares de las mujeres pobladoras, el movimiento antidictatorial de los derechos humanos, y el feminismo latinoamericano. De particular importancia para este texto, son los primeros años del grupo y el proceso de transformación que llevó a un tímido grupo de mujeres de "Iglesia”, amedrentadas por las condiciones cotidianas de la represión política y la escasez económica, a convertirse en una organización pionera en la lucha contra la violencia de género en Chile. Después de un breve resumen de este proceso, lo analizaremos desde algunas teorías de la ciudadanía y la acción colectiva, para profundizar nuestro entendimiento del significado de la Casa Yela en Chile. Luego, terminaremos este estudio con un par de reflexiones sobre el futuro de la Casa Yela, posterior a su consolidación en 1995, problematizando algunas dinámicas del grupo en relación al movimiento de las mujeres y al Estado.

\section{Cocinando solidaridad: las mujeres Yela durante los años '80 y '90}

Durante la década de los '60 llegaron cientos de miles de misionarios a Latinoamérica. Esto convergía, en el caso de algunas corrientes de la Iglesia Católica, con un nuevo enfoque en los pobres, manifestado en la teología de liberación y la educación popular. En el caso de Chile, llegaban cientos de mujeres de las Hermanas Maryknoll, una congregación católica estadounidense de mujeres religiosas conocida por su trabajo misionero en el mundo con comunidades pobres. Dos mujeres “Maryknoll”, Laura Magallanes y Jessie Poynton, llegaron a Chile en los años 50 y después se pusieron a trabajar en las poblaciones del sector norte de Talca en las décadas siguientes. ${ }^{1}$ Vivieron en dos mediaguas adyacentes en la misma pobla- 
ción y fueron muy activas en el desarrollo de los pobladores del sector, apoyadas por el Obispo Carlos González, quien buscaba acercar la iglesia a los sectores más pobres de Talca, tanto en las poblaciones urbanas como también en las zonas más rurales. ${ }^{2}$ González fue sucesor del Obispo Manuel Larraín, conocido por ser un gran promotor del Concilio Vaticano II en Latinoamérica y uno de los sacerdotes más vinculados a la fundación del Partido Demócrata Cristiano en Chile. Fue Larraín, en conjunto con el Arzobispo de Santiago Raúl Silva Henríquez, quien dio los primeros empujones a la Reforma Agraria en Chile, entregando un fundo del Obispado de Talca a los campesinos de la zona en 1962. Dos años antes, en 1960, Larraín escribió una declaración que evidenció su profunda creencia en la justicia social y la "preferencia para los pobres", una idea que compartió el mismo Obispo González: "Nuestro deber hoy para con Chile nos exige la defensa de la persona humana, la instauración de la economía orientada no hacia el lucro, sino hacia la satisfacción de las necesidades de todos los hombres, el respeto a la dignidad del trabajo en su hondo sentido humano y sobrenatural, conciencia aguda de que no vivimos el Evangelio mientras no rodeemos al trabajo del obrero y del empleado de la consideración que merece, la visión cristiana de una civilización orientada no hacia el "tener más”, sino hacia el "ser más” (citado en VVAA 2004:36).

La efervescencia social que se sentía en el campo con la Reforma Agraria también penetraba el sector conurbano de Talca, particularmente las poblaciones del sector norte de Talca. Fundadas a finales de los años 60 en gran parte como "tomas", fueron centros del poder popular y de participación en distintos partidos de centro-izquierda. Tal vez por esta misma razón, la represión después del golpe militar también se sintió con particular fuerza en este lugar. Como recuerda Lidia Riquelme, "Estabas durmiendo y te llegaban a allanar las casas [en] las poblaciones. Yo pololeaba con mi marido y vivía en población de campamento. De repente hacían levantarse a toda la gente que estaba durmiendo a título de nada o por buscar a una persona los ponían a todos en la calle acostados en el suelo y pasaban los militares por arriba de la gente. Los pisaban o los hacían salir a todos de las casas y los ponían en una cancha que hay aquí atrás". ${ }^{3}$ Este recuerdo es consistente con lo que señala un informe de Codepu sobre la represión en la región: “...las detenciones fueron masivas, realizadas tras operaciones de rastrillo en poblaciones campesinas o de copamientos militares en comunidades cordilleranas o precordilleranas. Todas las comisarías de las ciudades o de los poblados campesinos se encontraban repletas de personas que durante dos o tres días fueron sometidas a brutales técnicas de tortura" (Rojas 1996: 50). El Obispo Carlos González testimonia: "Visité la cárcel de Talca y me encontré con un hombre torturado, a quien colgaron de una viga con la cabeza hacia abajo para obtener información. Era un estropajo humano. Llegó otra persona con las manos quebradas por la tortura. El general de la zona me dice que se trataba de alguien que se autotorturaba para desprestigiar al gobierno” (González 2008: 25).

Por esta misma violencia, posterior al golpe militar del 73, las actividades de las hermanas Jessie y Laura se orientaban más hacia la 
concientización sobre los derechos humanos y la provisión de servicios básicos en la comunidad. Como parte de esta iniciativa, las hermanas también crearon un comedor popular en la capilla, adonde asistieron muchas mujeres pobladoras para ayudar en la preparación de las comidas. María Teresa Riquelme, una de las mujeres Yela y muy cercana a las hermanas , recuerda: “...ellas hicieron los comedores donde se cocinaba la olla común en la Iglesia y la gente iba a buscar su comida, iban los niños, ancianos a almorzar, la gente iba a buscar su comida, los ancianos a almorzar, ellas trabajaron mucho con esa olla común y también llegaba mucha ayuda de afuera también por intermedio de Caritas, cuando ellas estuvieron acá hubo mucha ayuda, llegaba harina, leche, llegaban muchos alimentos y llegaba ropa donde ellas distribuían todo eso (...) fueron ellas muy, pero muy solidarias.” ${ }^{4}$ Fue durante la preparación de las ollas comunes que las mujeres empezaban a conversar más sobre sus vidas personales y las hermanas empezaban a observar mujeres que llegaban con señales del abuso físico, por ejemplo los ojos morados, moretones corporales y el estado anímico bajo; algunas mujeres se ponían a llorar y hablaban de los abusos que padecían en sus casas. Para abrir un espacio de reflexión, las hermanas decidieron invitar algunas mujeres a venir a la capilla y ver una película - El Color Púrpura de 1985. Sería un evento que marcaría profundamente a las vidas de las mujeres que posteriormente se integrarían a la Casa Yela - todas se acuerdan de esta película y la manera profunda en que fueron tocadas por su historia de violencia doméstica y sexual.

Para los años 80, el movimiento feminista internacional ya había empezado a organizar en torno la problemática de la violencia contra la mujer - la primera casa de acogida para las mujeres golpeadas fue abierta en Inglaterra en 1971, seguida por casas similares en EEUU y otras partes de Europa - , y la ONU además ya había pasado la Convención sobre la Eliminación de Todas Formas de Discriminación Contra la Mujer, o CEDAW, en 1979. Las Hermanas Maryknoll fueron pioneras en el trabajo de este tipo en Chile, inspiradas por la influencia del feminismo en el mundo secular y las lecturas de mujeres religiosas que tocaban temas relacionados al género. Como señala Jessie Poynton sobre este período: "Se estaba cambiando la vida en Chile. En Santiago, se abrían centros de empoderamiento y de salud mental para las mujeres. Nuestras hermanas abrieron Casa Sofía y Casa Malén en las poblaciones del sector norte de Santiago. Nosotras también aprendimos de ellas. Nos informamos y seguimos algunos de sus programas, utilizando las dinámicas de grupo que habían tenido éxito con las mujeres de las poblaciones santiaguinas." 5

Como parte de la preparación de las mujeres talquinas, a comienzos de los años 80 algunas mujeres acompañaron a Jessie y Laura a un encuentro de mujeres pobladoras en Santiago que tenía lugar en el Colegio Andacollo. Ahí las mujeres “de iglesia” de Talca empezaban a ver que las mujeres pobladoras también podían trabajar temas que se asociaban con el feminismo. Como recuerda Leonarda Gutiérrez: "Y ahí nos quedamos espantadas con los temas que se trataban porque éramos mujeres de Iglesia. 
Nos creíamos mujeres de la Iglesia. Íbamos con rosario y con testamento para esa jornada. Y cuando llegamos allá decía, "Primer Encuentro Feminista no sé cuanto” (risas). Y entráramos e inscribíamos y nos entregaron carpetas con el programa de todos los talleres que habían. Y no encontramos lo que nosotras más-menos buscábamos. Pensábamos que íbamos a encontrar un letrero como "aprender a usar la Biblia”, esas cosas. Y nos encontrábamos con "Taller de Aborto", “Taller de Anti-conceptivos”, hasta que ahí al final encontramos el taller de violencia.....Nos encontrábamos ignorantes, ignorantes de muchos temas. Nos decíamos - ¡tantos temas y la facilidad con que hablaban y tan abiertas! - en ese sentido me encontré ignorante". ${ }^{6}$ Al volver a Talca, las mujeres empezaban a organizarse más para hablar explícitamente de la violencia y el empoderamiento de las mujeres. Las hermanas pasaban temas con un grupo reducido de mujeres y después este grupo fue invitando a otras pobladoras para enseñarles lo que habían aprendido. Fue durante este período, además, que este grupo de mujeres talquinas tuvieron sus primeras capacitaciones con mujeres de la Casa Malén (otro proyecto producto de las Hermanas Maryknoll pero en Santiago) - con Rogelia Molina y Margarita Suárez - que hasta el día de hoy son recordadas con mucho afecto y que les ayudaron con información y tácticas de organización. Para el año 1986, las mujeres de lo que sería la Casa Yela ya habían estado trabajando hacía varios años la temática de la violencia contra la mujer y estaban listas para manifestarse públicamente. La primera directora de la Casa Yela, Elena Valenzuela, recuerda así la salida pública del grupo: "Dijimos, “iVamos a salir a la calle con estas mujeres!”; primera vez en Talca, no pedimos ni permiso primero a las autoridades... en ese tiempo, difícil. Dijimos, "Nos vamos a venir por todas las calles centrales de Talca desde la 11 oriente, desde el terminal hacia abajo hasta la Plaza de Armas”... Nos conseguimos más poleras y nos pusimos estas poleras, hicimos pancartas -muy mal hechas porque no habían recursos tampoco- hicimos gritos, hicimos cosas alusivas con nuestra propia imaginación y nos vinimos por la calle principal de Talca. Oye...jnos tiraron piedras! (habían construcciones en el centro de Talca); los dueños de las casas comerciales se paraban en la puerta, nos decían "mujeres locas”, “ ¡vayan a hacer el almuerzo!”,” tontas” y muchas más cosas que ésas y los Carabineros, mucho más represivos que ahora, nos echaban a la vereda; nosotras nos bajábamos [a la calle].” 7

Después de esta fecha, las mujeres del grupo formaron su primera casa de acogida, con espacio para apenas dos personas, en Villa la Paz y siguieron juntándose con mujeres capitalinas para avanzar en sus capacitaciones sobre la temática de la violencia de género. Entremedio, fueron también activas en el movimiento antidictatorial y participaron en una iniciativa ciudadana para "enseñarles” a votar a las mujeres para el plebiscito. No obstante, los años 1989-90, serían bien difíciles para estas mujeres porque fue un período cuando las Hermanas Maryknoll, tan cercanas a ellas, se tuvieron que ir. Las hermanas, bastante progresistas y feministas en su orientación, habían llegado a un punto de tope en su misión en Chile. El Obispo de Talca, Carlos González - tan solidario y progresista en temas de los derechos humanos y los derechos sociales - no aceptaba el trabajo "fe- 
minista” de las Hermanas Maryknoll y su cercanía con el movimiento de mujeres. Además, el tema de la violencia doméstica y sexual fue considerado como potencialmente peligroso por el obispo, ya que podía provocar el fracaso de los matrimonios. Como señala Leonarda Gutiérrez: “[González] Cuestionaba los temas, porque decía que... nosotras habíamos empezado a separar el matrimonio, al tocar los temas de violencia, empezar a descubrir, hacer a la mujer tomar conciencia de lo que estaban viviendo y que no tenían que seguir aguantando. Pero él lo tomaba como que nosotras estábamos separando los matrimonios, que íbamos a armar más polémicas en los matrimonios" . A pesar de que las mujeres "Yela" aseguraban que esto no fue el caso, y que en realidad sólo querían contribuir a matrimonios más sanos e igualitarios, se había producido una ruptura importante entre la Iglesia y el proyecto de las mujeres Yela. Las Hermanas, con mucho pesar, tuvieron que buscar una nueva misión en Chile y volvieron a Estados Unidos a principios de los 90 .

Salir del espacio protegido de la Iglesia les trajo serios problemas en términos organizacionales. En primer lugar, las mujeres Yela se encontraron sin los fondos necesarios para mantenerse, ya que las Hermanas Maryknoll habían sido nexos importantes en ese aspecto. En segundo lugar, y por estos mismos problemas financieros, ya no podían seguir arrendando la casa de acogida en la villa y no tenían donde reunirse. Fue un período cuando las mujeres Yela, sin recurso alguno, tuvieron que recurrir a una plaza pública para sus reuniones. Pero incluso durante este período tan difícil no se dejaron de juntar de manera regular ni tampoco se olvidaron de lo que habían aprendido con las hermanas. Guacolda Saavedra lo recuerda así: “...cuando las hermanas recién se fueron, nosotros quedamos a brazos cruzados, sin ningún financiamiento. En ese momento tuvimos que guardar las cosas en la casa de una de las compañeras y hacíamos igual las reuniones en la plaza del sector de Villa La Paz. Igual nos reuníamos el mismo día que habíamos dejado instalado como día de reunión y nos juntábamos ahí. Como no teníamos financiamiento, [y ]como en mi caso que yo era de este sector, yo iba en bicicleta a mis reuniones" ${ }^{9}$. Justo en este momento de más necesidad, la Casa Yela logró aprovechar las redes nacionales que tenía con grupos feministas y empezó a surgir de nuevo. En 1991, las mujeres "Yela” participaron en el Primer Encuentro de Violencia en el país en Valparaíso. Durante esta actividad ellas pudieron compartir con mujeres de todo Chile y del extranjero - incluyendo las de la recién formada Red Chilena contra la Violencia Doméstica y Sexual a la cual se afiliaron - y el líder del grupo, Elena Valenzuela, conoció a Ana María Órdenes, del grupo SOL (Solidaridad y Organización Local) por primer vez. Este encuentro fue bastante fortuito, ya que con la ayuda de SOL, que recibía fondos desde Europa para organizaciones femeninas de base, la Casa Yela pudo organizar pequeños proyectos y actividades y se activaron como miembros de una comunidad de mujeres que trabajaban a nivel nacional. Después de estos primeros talleres financiados por SOL, las mujeres Yela decidieron formalizarse como monitoras en violencia doméstica y sexual, cursando una serie de capacitaciones con la ONG feminista santiaguina La Morada. 
Como parte de esta nueva fase organizacional, las mujeres de la Casa Yela lograron un sueño del grupo y pudieron conseguir fondos de una ONG suiza para fundar una buena casa de acogida con espacio para talleres y actividades del grupo. Esto se produjo por la iniciativa de Elena Valenzuela y su cercanía con el diputado socialista Sergio Aguiló, quien, en conjunto con la diputada PPD Adriana Muñoz, fue autor de la primera ley de violencia intrafamiliar. Cuando Valenzuela lo presionó para una casa, Aguiló contactó a Adrián Solar, un productor de cine que había vivido muchos años en Alemania y que conocía gente en la ONG suiza Terre des Hommes, y así las mujeres Yela fueron preparando la mayor propuesta de su vida organizativa. Sobre esta secuencia de eventos Valenzuela, narra lo siguiente: “...vamos a ir a la oficina de Sergio, y como yo lo conocía de antes, no como Diputado, [y] era muy amiga de la secretaria, no necesitaba ni audiencia. Le dije, “¿Sabes? necesito hablar con el Diputado”...Le dije [a Aguiló], "Usted publica en su cuenta parlamentaria que usted consiguió una casa a la Mujer en Talca y eso es una gran mentira. Nosotras no tenemos arriendo, no tenemos donde estar, no tenemos como pagar luz, como pagar agua... [él] pedía disculpas y...entonces me dijo, "Vamos a solucionarlo”. Llamó a una persona y me dijo, "Las va a recibir a las cuatro de la tarde.” Esta persona era un ex-talquino que estaba radicado en Alemania mucho tiempo... y vamos donde este Señor y nos recibe en la tarde, que era don Adrián Solar. Él dice, “A ver, ¿qué pasa?” Y le contamos. Entonces, nos dice, “¿Ustedes son capaces de realizar de aquí a mañana un proyecto?” Corrimos para allá y para acá -incluso recuerdo que me di un golpe en esa oportunidad sacando fotocopias; me hice tira las rodillas- a las diez se lo teníamos”10. Aunque habían empezado a arrendar esta casa con ayuda de SOL, con el financiamiento del extranjero pudieron montar un proyecto de mayor envergadura - comprando la casa en Villa las Palmas y formulando un programa de financiamiento que duraría para un período de aproximadamente diez años. En 1995 nació formalmente la Casa de la Mujer "Yela" (en honor a "YE-ssica” y "LA-ura”), como un lugar conocido de refugio y de organización feminista en Talca.

\section{Movimientos sociales, ciudadanía, y la "nosotras" colectiva de la Casa Yela}

Durante los años ochenta, era bastante común dentro de la literatura sobre los movimientos sociales en Latinoamérica encontrar conclusiones que proponían que éstos estaban creando un nuevo espacio democrático y participativo en la región. Teóricos críticos de la ciudadanía y la democracia buscaban promover el concepto de una ciudadanía activa como base para la democratización de las sociedades latinoamericanas y, como bien señala Maxine Molyneux: "Las analistas feministas centraron su atención en otorgar visibilidad y valor a la participación femenina, mientras debatían sobre el carácter generalizado de las formas de movilización y las reivindicaciones que la acompañaban...La democracia se concebía no sólo como una práctica de política institucional formal, sino como una práctica 
que afectaba la vida cotidiana e impregnaba la familia y la sociedad en general. Ello implicaba redefinir el significado mismo de la democracia" (Molyneux 2003: 273-4). Dentro de Chile, el tema de la democratización, tanto del país como de las relaciones de género, se ve con claridad en los trabajos de mujeres como Julieta Kirkwood, Teresa Valdés, y Marisa Weinstein, todas académicas de Flacso durante la dictadura. Estas últimas, Valdés y Weinstein, fueron dos voces importantes, además, en la discusión sobre la participación de las mujeres pobladoras en las luchas anti-autoritarias y feministas, proponiendo que la manera "pobladora" de hacer política - sus prácticas y saberes - también debía tener un lugar en la nueva democracia (Valdés 1988, Valdés y Weinstein 1993, Weinstein 1996). Pero no fueron sólo mujeres preocupadas de temas de género las que reivindicaban la participación democrática desde abajo (desde los grassroots). Académicos, como Mario Garcés (2002), Philip Oxhorn (1994, 1995) o Cathy Schneider $(1991,1995)$, también concluían que la exclusión de los sectores populares y las organizaciones pobladoras de la participación en los partidos políticos y, más generalmente, del sistema de representación política, podía clausurar la posibilidad de instalar una verdadera democracia en Chile.

Este tipo de reflexión sobre la democracia, necesariamente nos lleva a interpretaciones más "plenas" de la ciudadanía, similar a lo que señala Chantal Mouffe sobre la ciudadanía “demócrata-radical”, que: “enfatiza las numerosas relaciones sociales en las cuales existen situaciones de dominación y que tienen que ser desafiadas para que los principios de libertad y igualdad puedan ser aplicados. [Esta definición] indica que el reconocimiento compartido por parte de los distintos grupos que luchan para una extensión y una radicalización de la democracia tiene que ver con una preocupación en común. Esto debe desembocar en la articulación de las demandas democráticas encontradas en una serie de movimientos: mujeres, obreros, negros, homosexuales, ecológicos, y también los "nuevos movimientos sociales". La meta es construir un "nosotros" como ciudadanos democráticos radicales, una identidad política colectiva articulada a través de los principios de la equidad democrática, aunque se debe destacar que una relación de equidad no elimina la diferencia...” (Mouffe 1992: 378-9). En términos de acción colectiva, los individuos, según Alberto Melucci, también crean un "nosotros” colectivo que tiene que ver, a grandes rasgos, con tres tipos de orientaciones: (1) las que se asocian con los fines de la acción; (2) las que tienen que ver con los medios de la acción; y (3) las que se sitúan dentro de los límites del contexto socio-histórico (Melucci 1997). Si pensamos en la "nosotras" de la acción colectiva de la Casa Yela del período 1980-1995, también podemos hacer un análisis según estas tres orientaciones para tres períodos claves. En primer lugar, entre aproximadamente 1980 y 1985, los fines y los medios de la acción de este grupo de mujeres claramente dependían de una reflexión sobre la violencia en sus comunidades que tenía que ver con el contexto dictatorial. Acompañadas por las Hermanas Maryknoll, actoras claves durante la formación de lo que sería la plataforma principal de la Yela, las mujeres Yela empezaban a concentrar sus esfuerzos organizativos en el tema de la violencia contra la mujer, algo poco común para el período. Esta nueva orientación hacia la violencia 
también les iba a transformar; desde ser mujeres de “iglesia”, preocupadas de la catequesis y el cuidado "cristiano" de los ancianos y los niños, las mujeres "Yela” se volvieron más feministas en su cosmovisión y empezaron a vincular la violencia con la organización patriarcal de sus familias y de la comunidad. Después de contactos facilitados por las hermanas Jessie y Laura, estas mujeres talquinas iniciarían intercambios con mujeres santiaguinas que les van a capacitar sobre numerosos temas de empoderamiento y violencia. Esto es un período de definición y de concientización que muchas mujeres Yela recuerdan como un gran “despertar” y que podemos relacionar muy cercanamente con el concepto de “capital social” formal de Robert Putnam, ya que las mujeres utilizaban sus redes personales y comunitarias para formar lo que llegaría a ser una organización social. ${ }^{11}$

Durante el segundo período, de 1986 y 1990, las mujeres Yela comienzan a destacarse como organizadoras en su comunidad y como figuras pioneras en la lucha contra la violencia de género. El 25 de noviembre de 1986 las mujeres Yela aparecen públicamente por primera vez en Talca, protestando contra la violencia de género en un contexto difícil. Sólo dos meses antes, el 7 de septiembre de 1986, se había cometido el atentado contra el General Pinochet en el Cajón del Maipo, en las afueras de Santiago, y el gobierno había declarado un estado de sitio en todo el país y aumentado el nivel de represión acorde con sus planteamientos de la Seguridad Nacional. ${ }^{12}$ Como Elena Valenzuela recuerda, muchos hombres de la calle les lanzaban insultos y algunos llegaban a tirarles piedras; además, fue durante un contexto dictatorial cuando las marchas frecuentemente podían terminar en violencia y represión por parte de la policía. Pero ellas se mantenían firmes y lograron abrir su primera casa de acogida en Villa la Paz, ayudadas por lo que recibían de las Hermanas. Esta situación cambió al entrar a los noventa, cuando se van Jessie y Laura, quedando las mujeres Yela, en buen chileno, "sin pan ni pedazo". Esto les pone en la situación incómoda de ser un grupo más o menos consolidado, pero sin un lugar físico propio de acción. Algunas mujeres no resisten y se van, pero otras siguen adelante, juntándose en la plaza e incluso a través de un encuentro nacional se insertan más en la red del movimiento de las mujeres (con sede principal en Santiago), logrando salir adelante y montar proyectos de nuevo. El sentido de ser "luchadoras" se profundiza, ya que enfrentan numerosos obstáculos en Talca, relacionados no sólo a la falta de financiamiento y su estatus como mujeres populares, sino también a la dificultad de trabajar temas de género en provincia y a la hostilidad de la Iglesia Católica frente sus temas (que fueron entendidos por algunos sacerdotes como "antifamilia” o “anti-naturales”). Se visualiza la necesidad de poder levantar una nueva casa de acogida y entablar sus demandas con más fuerza desde este lugar, definiéndola como una prioridad del grupo en este periodo.

Para el tercer período de estudio, 1991-1995, las mujeres Yela participaron en proyectos que las visibilizaron en la comunidad y a un nivel nacional. Por ejemplo, realizaron unas encuestas en Talca sobre la violencia doméstica que fueron claves en la formulación de la primera ley de 
violencia intrafamiliar de 1994. También trabajan más de cerca con organizaciones santiaguinas, como SOL, la Red contra la Violencia Doméstica y Sexual, y La Morada, este último capacitándoles como monitoras de violencia, etapa clave para "profesionalizarse” en esta materia. Además, a través de un lobby acertado con el diputado Aguiló, logran comprar su propia casa de acogida. Visto desde la perspectiva de un grupo que, en algunos momentos duros, se tenía que reunir en una plaza pública, haber conseguido la casa significó concretizar un sueño colectivo del grupo.

Desde 1995 en adelante, la Casa Yela formulará políticas y programas desde un lugar más institucionalizado y profesionalizado en el escenario local y nacional. Con la ayuda del trabajador social Marcelo Pinochet, entre 1995 y 2000 la Casa Yela se “abre” a un público más general y se inserta estratégicamente dentro de las lógicas del Estado, formando alianzas temporarias con distintas instituciones. Las mujeres Yela capacitan a funcionarios de los Carabineros y del sistema público de salud, hacen actividades de prevención y concientización en los colegios y jardines infantiles, y plantean temas de violencia dentro de discusiones locales sobre la seguridad ciudadana. Además, la Casa Yela mantiene, durante este período, una relación relativamente positiva de trabajo con SERNAM, participando en un comité y en distintas actividades sobre la violencia contra la mujer. En el plano de la justicia, las mujeres Yela se transforman en interlocutoras importantes de la zona, movilizándose en protesta frente el femicidio de Isabel González en 1997 - quemada por su conviviente - y el ataque brutal perpetrado contra Marcela Valenzuela por su tío con una motosierra - que le cortó una mano - en el 2002. Estos dos crímenes escandalizaron la zona, llegando a ser noticias de primera página, y situaban a las mujeres Yela como expertas en la violencia contra la mujer dentro del contexto local y nacional. La fama y el buen nombre de la Casa Yela y sus miembros estaban a tal nivel que en el 2000, dos monitoras -Leonarda Gutiérrez y Benedicta Aravena- fueron invitadas a participar en actividades internacionales sobre la violencia de género, la primera en un encuentro latinoamericano sobre violencia de género y casas de acogida en Nicaragua, organizado por el BID; y la segunda en la marcha mundial por las mujeres contra la violencia y la pobreza (marcha "Pan y Rosas") en Estados Unidos.

Hasta este punto, podríamos decir que hemos trazado una línea más o menos "esperanzadora” sobre la trayectoria de la Casa Yela en su relación con la democracia y la ciudadanía en Chile. A través de su lucha como parte de los movimientos sociales de los años 80, las mujeres "Yela” lograron hacer algo que muy pocos grupos pudieron hacer - establecer su propia casa de acogida y obtener un financiamiento estable para la casa durante una década. Para cualquier activista feminista que vivió estos años, la Casa Yela fue casi una leyenda; una muestra ejemplar de lo que las mujeres populares podían lograr y del poder del movimiento. Sin embargo, si trasladamos nuestro marco histórico hacia la última década, 2000-2010, llegaríamos a conclusiones muy distintas sobre la Casa Yela ${ }^{13}$. Sin profundizar demasiado el argumento, es relevante señalar que pareciera que todo 
lo que había sido el soporte financiero y organizacional de la Casa Yela entra en crisis. El financiamiento externo de las ONGs internacionales llega a su fin porque Chile ya no es considerado un país "tercermundista" tan necesitado. El financiamiento interno del Estado no les ayuda porque SERNAM decide reemplazar las casas de acogida mantenidas por ONGs con casas propias del Estado. Y el movimiento de mujeres, por lo menos en términos de su conexión con las mujeres populares, tiene las manos amarradas, "castigado" por los mismos problemas financiamiento. Para el año 2010, la Casa Yela ya no puede acoger a las mujeres como podía hacer anteriormente y se gira hacia un perfil de centro comunitario, administrado por un grupo de mujeres. De esta manera, la Casa Yela sigue en pie y atendiendo a mujeres populares del barrio, pero con una orientación distinta en términos de los servicios proveídos (o por lo menos es así al momento de la publicación de este artículo).

\section{Un modo de concluir: las estrategias cambiantes de la Casa Yela y los límites de la acción colectiva dentro del Estado neoliberal}

Si miramos la trayectoria de la Casa Yela durante los años 19801995, nos llama la atención el dinamismo de las mujeres Yela en su diseño de distintas estrategias que buscaban resguardar el funcionamiento del grupo a través de los años. Durante el primer período del grupo, 1980-1986, las mujeres Yela tuvieron que enfrentar retos organizacionales que correspondían principalmente a la etapa formativa de un grupo, transformándose en mujeres activistas en torno a la temática de la violencia contra la mujer. En este trabajo de definición las hermanas Maryknoll fueron decisivas; ayudaron a orientar el grupo hacia actividades específicas, traían materiales desde la capital y desde el extranjero, e integraron un discurso teológico sensible a las necesidades de las mujeres populares. Por el contexto histórico de Chile en ese momento, las mujeres Yela tenían una especial cercanía con la Iglesia que les proveía de ciertos recursos, como comida para el comedor popular y un lugar de reunión en la parroquia. Además, les “cobijaba", en el sentido de darles un espacio relativamente "seguro" dentro de lo cual podían trabajar y enunciar sus necesidades. Esto dista mucho de su relación con el Estado, que fue visto como represor e injusto. El régimen autoritario de Pinochet no les ofrecía ningún espacio de participación política - aparte de ser "socias" dentro de programas paternalistas y clientelistas, como CEMA-Chile, liderado por Lucía Hiriart -y castigaba brutalmente a los sectores populares en términos políticos y socio-económicos. Las mujeres Yela recuerdan los numerosos allanamientos e intervenciones militares en las poblaciones y asocian este período con la extrema pobreza y la miseria. La acción colectiva en este contexto, en particular la formación del comedor popular y la olla común, se erige como resultado lógico de las circunstancias autoritarias y como fuente de las reflexiones que motivarían al grupo durante sus etapas posteriores, consolidando en ellas su carácter de "luchadoras" sociales y solidarias que podían intervenir positivamente 
en su comunidad. En ese sentido, aunque rescatamos la observación astuta de Maxine Molyneux (2003) que no todas las ollas comunes de los años ochenta desembocaron necesariamente en el empoderamiento de las mujeres en Latinoamérica, en el caso de las mujeres Yela en Talca eso fue precisamente lo que pasó.

Este aspecto de la acción colectiva es compartido por el movimiento de mujeres de los años 80 en Chile que desembocará en 1990 en la elección de Aylwin y la vuelta a la democracia. Desde 1986 en adelante, prácticamente todos los movimientos sociales, incluyendo el de las mujeres y de las feministas, se vuelcan hacia la proyección del fin de la dictadura y la vuelta a la democracia. Lo que en algún momento, aproximadamente entre los años 1980-85, fue sólo una vaga posibilidad, se empezó a cristalizar con el fallido atentado contra Pinochet y la decisión por parte de la oposición de dirigir todas sus energías hacia el plebiscito de 1988. Dentro de este panorama, las mujeres Yela también empiezan a integrarse dentro de un movimiento más amplio, tanto de mujeres feministas (conferencias, foros, talleres) como de mujeres antidictatoriales (enseñanza de voto). Sin embargo, posterior a las victorias del "No" y de Aylwin, la reintegración de los partidos políticos al sistema nuevamente democrático va a alterar radicalmente las estrategias de las mujeres Yela. Esto es complicado, además, por la salida de las hermanas Jessie y Laura de Talca y el correspondiente fin de su cercanía con la Iglesia. Ahora ellas dejan de juntarse en la parroquia y empiezan a reunir en la plaza, con todo el simbolismo que este cambio conlleva (desde el espacio "protegido" de la comunidad eclesiástica al lugar público y "peligroso" de la plaza). Dentro de esta coyuntura, las mujeres Yela son capaces de reconocer un posible aliado en el diputado socialista Sergio Aguiló y lo presionan con éxito para que él les ayude a conseguir una solución a su problema (estar sin casa). Así, Aguiló mueve sus redes personales y logra conseguir que ellas postulen a Terre des Hommes, con un proyecto ambicioso. Al ganar el apoyo de esta ONG suiza, las mujeres "Yela” obtienen una fuente segura de financiamiento, pero también entran en los vaivenes, a veces muy volátiles, de la “ongeización” internacional (Alvarez 1998, Keck y Sikkink 1998). Además, como ya tienen una casa y un financiamiento seguro, ellas no tienen que preocuparse de mayor manera de su relación con el Estado, ya que pueden operar de manera relativamente autónoma. Se consolida una relación más fuerte con otras ONGs feministas de mujeres, tanto de la capital como de otras regiones, y las mujeres Yela se destacan como mujeres pioneras y expertas en su tema. Este reconocimiento llega a tal nivel que el grupo es incluido dentro de proyectos específicos del Estado - como "consultoras” y "capacitadoras" independientes - en programas sobre la violencia doméstica y sexual asociados a los rubros de salud, educación, y seguridad ciudadana. Ellas disfrutan de una visibilidad pública en los medios de comunicación locales y se vuelven interlocutoras legítimas en los debates sobre la violencia y la mujer.

Tal vez por este mismo éxito tan pronunciado, van a ser mayores las desilusiones del grupo a partir del año 2005, cuando el financiamiento de 
Terre des Hommes termina definitivamente. Las estrategias que les habían servido anteriormente - principalmente activadas a través de vínculos personales con actores claves que les conectaban con fuentes poderosas de financiamiento (la Iglesia Católica y una ONG internacional) - ahora parecen ser inoperantes. Al comienzo de nuevo milenio, Chile ya no es considerado una prioridad para el financiamiento de las ONGs dedicadas al "tercer mundo", y la Iglesia, por lo menos en Talca, no tiene una preferencia tan marcada para los pobres y justicia social. Además, el giro hacia el feminismo les clausura la posibilidad de volver a ser mujeres "obedientes" de iglesia en lo que concernía sus discursos sobre la mujer y la familia. Así, las mujeres Yela se quedan sólo con el Estado, el mismo ente que ofrecía servicios muy similares a través del SERNAM, y que tenía pocas razones "racionales" (dentro de un modelo tecnocrático y neoliberal) para querer financiar a una organización de "competencia”. Pero la erosión de interés estatal no es sólo producto de este solapamiento de servicios, sino que corresponde también a una reducción generalizada en el interés de promover la participación de las bases (léase sectores populares) en la política y la desarticulación paulatina y permanente de los movimientos sociales en el escenario socio-político del país. Las mujeres Yela son “desechables” dentro de la democracia neoliberal y la sociedad de consumo de Chile, un hecho que es percibido por ellas como una "traición” de los valores democráticos prometidos en el plebiscito de 1988. Y aunque podríamos decir que esta traición fue más que predecible dado la historia socio-política del país, ese tipo de justificación fácil no les puede quitar a las mujeres Yela la amarga sensación de la marginalización. Como dice la monitora Margarita Oyarzún, que lleva años trabajando en la Casa Yela: "Para mí, ahora la Casa Yela es decir "¿de dónde yo saco o a quién pedirle ayuda para que esto siga funcionando?, porque esto no se puede cerrar por el prestigio que tiene la Yela por los años de trabajo que lleva. Por el servicio que ha prestado montones de años a esta comunidad, a este Talca, a Chile entero porque hemos tenido gente de todas partes de Chile. Y el sueño dorado es que esto siga funcionando que esto no se pierda por no tener subvención, que siga, que esto no se pierda. Que se vaya a cerrar...pienso que sería una pérdida enorme, enorme para la nación." ${ }^{14}$ 


\section{Notas}

* Este artículo forma parte de la investigación de mi tesis doctoral, "Historizar la violencia puertas adentro. El caso de la Casa Yela y la violencia de género en Talca, 1964-2008”. Se realizó gracias a la beca Conicyt "Realización de Tesis Doctoral” 2009-2010 y la beca MECESUP “Beca en el Extranjero para Doctorandos en Tesis” 2010. Fue presentado originalmente como ponencia en el simposio “Acción colectiva y Movimientos sociales” en el Congreso Ciencias, tecnologías y culturas. Diálogo entre las disciplinas del conocimiento. Mirando al futuro de América Latina y el Caribe que se llevó a cabo en la USACH, entre el 29-31 de octubre de 2010. Les agradezco a mis colegas en la Universidad Diego Portales, Juan Pablo Paredes y Carlos Durán, quienes organizaron este simposio y me invitaron a participar. Agradezco, además, y con todo mi corazón a las mujeres Yela y, en particular, a Leonarda Gutiérrez. Sin ellas este proyecto no habría sido posible.

${ }^{1}$ Como dice en la página web de las Hermanas Maryknoll: "Maryknoll Sisters were invited by the Cardinal Archbishop of Chile to work for the education of the very poor. The Maryknoll priests and brothers also extended an invitation to collaborate with them in their parish schools. The first two Maryknoll Sisters went to Galvarino in 1950, and the pastor wrote: "God sent them.” The Maryknoll Sisters won the hearts of the people...One hundred and three Maryknoll Sisters have served in Chile from the far south to the desert north. Those first 15 years were a fruitful time of staffing schools for the poor. As an aftermath of the Second Vatican Council, there was a shift from institutions to direct evangelization with the Chilean Church. Chilean bishops desired a strong Church presence in the marginated areas of the larger cities. The basic option for the poor didn't change but a new way of sharing life with the poor, learning daily from them and serving them, has developed. Justice issues and the defense of human life permeated all the ministries.” (http://www.mklsisters.org/index.php/ component/gmapfp/print_article/component/8?flag=1).

${ }^{2}$ La séptima región se tipifica por ser una zona agraria, históricamente marcada por el legado de los latifundios y los grandes terratenientes. "En 1970, más de la mitad de la población era rural, 325.015 habitantes (52,64\%), siendo el sector con mayor protagonismo social y político entre ese año y mediados de 1973. La expropiación de fundos, las tomas de terrenos y el aumento de la organización campesina se hicieron frecuentes desde fines de la década del 60" (Rojas 1996: 35).

${ }^{3}$ Lidia Riquelme, Entrevista personal, Talca, 8 de febrero de 2010.

${ }^{4}$ María Teresa Riquelme, Entrevista personal, Talca, 19 de febrero de 2010.

${ }^{5}$ Jessie Poynton, Email personal (traducido por la autora), 25 de febrero de 2010.

${ }^{6}$ Leonarda Gutiérrez, Entrevista personal, Talca, 13 de diciembre de 2008.

${ }^{7}$ Elena Valenzuela, Entrevista personal, Talca, 15 de febrero de 2010.

${ }^{8}$ Leonarda Gutiérrez, Entrevista personal, Talca, 15 de enero de 2010.

${ }^{9}$ Guacolda Saavedra, Entrevista personal, Talca, 12 de febrero de 2010.

${ }^{10}$ Elena Valenzuela, Entrevista personal, Talca, 15 de febrero de 2010. 
${ }^{11}$ El sujeto social maneja una red de relaciones personales y profesionales que utiliza para tomar medidas en distintas circunstancias. Durante momentos decisivos de la vida (lo que Giddens llama, "fateful moments" ) o de guerra/represión política, las opciones posibilitadas por estas redes puedan llegar a tener consecuencias dramáticas en la vida de un individuo o un grupo social tal. Se puede hablar, entonces, de las formas múltiples de capital social que cada individuo tiene a su disposición como individuo, y como miembro de varios grupos, en una sociedad. Algunos sujetos optarán por la utilización de un capital social más "formal”, como unirse a una organización tipo "nuevo movimiento social” o a un sindicato, una iglesia, etc. Otros por las redes y relaciones más relajadas (llamadas "loose relations" por Wurthnow en el contexto estadounidense), que tipifican el capital social “informal” (Putnam 2002).

${ }^{12}$ La fecha de la aparición de la Casa Yela es, hasta cierto, punto un poco anómala en el contexto chileno, ya que a partir de los fines de 1986 hay un "verdadero eclipse" de los movimientos sociales en Chile, según Patrick Guillaudat Pierre Mouterde (1998: 174), ocasionado por el ambiente represivo del post-atentado y la decisión, por parte de los partidos políticos, de concentrar sus esfuerzos en el desarrollo de un consenso político para el plebiscito de 1988. Al contrario del período 1981-1985, los finales de los ochenta se tipifican por el lento repliegue de los movimientos sociales y el renacimiento del Estado como principal locus de acción. Sin embargo, por otro lado, en términos de lo que pasaba dentro del movimiento feminista respecto la violencia contra la mujer, las fechas se coinciden con más facilidad, ya que desde el año 1986 hay más movilización y protesta sobre los derechos de la mujer y la violencia contra ella.

${ }^{13}$ De este tema se trata un capítulo de libro escrito por la autora, "Mujeres entre "la espada y la pared”: violencia de género y Estado en Chile, 1990-2010” (en prensa), presentado parcialmente como ponencia en la mesa "Nuevas ciudadanías: tensiones y disputas en el Chile del Bicentenario" (Coordinadora: Consuelo Figueroa; Discussant: Heidi Tinsman) del "XXIX Congreso Internacional LASA" en Toronto, Canadá.

${ }^{14}$ Margarita Oyarzún, Entrevista personal, Talca, 29 de septiembre de 2008. 


\section{Bibliografía}

Alvarez, Sonia (1998), “Latin American Feminisms Go Global: Trends of the 1990s and Challenges for the New Millenium”, en Sonia Alvarez, Evelyn Dagnino y Arturo Escobar (eds.), Cultures of Politics/Politics of Culture, Westview Press, Boulder, pp. 293-324.

Garcés, Mario (2002), Tomando su sitio. El movimiento de pobladores de Santiago, 1957-1970. LOM, Santiago.

Guillaudat, Patrick y Mouterde, Pierre (1998), Los movimientos sociales en Chile, 1973-1993, LOM, Santiago.

González Cruchaga, Carlos (2008), ¿Y qué hiciste con tu hermano? Testimonio de un obispo, 1973 a 1990, LOM, Santiago.

Keck, Margaret y Kathryn Sikkink (1998), Activists Beyond Borders. Advocacy Networks in International Politics, Cornell University Press, Ithaca.

Melucci, Alberto (1999), Acción colectiva, vida cotidiana y democracia, Colegio de México, México.

Molyneux, Maxine (2003), Movimientos de mujeres en América Latina, Cátedra/Universitat de Valencia/Instituto de la Mujer, Madrid.

Mouffe, Chantal (1992), "Feminism, Citizenship and Radical Democratic Politics”, en Judith Butler y Joan Scott (eds.), Feminists theorize the political, Routledge. New York, pp. 369-383.

Oxhorn, Philip (1994), "Where did all the protesters go?: Popular mobilization and the transition to democracy in Chile”, en Latin American Perspectives Vol. 21, No. 3, pp. 49-68.

Ídem (1995), Organizing Civil Society. The Popular Sectors and the Struggle for Democracy in Chile, Penn State Press.

Putnam, Robert (ed.) (2002), Democracies in Flux. The Evolution of Social Capital in Contemporary Society, Oxford University Press, New York.

Rojas, Paz (coord.) (1996), Crímenes e impunidad. La experiencia del trabajo médico, psicológico, social y jurídico en la violación del Derecho a la Vida. Chile 1973-1996. VII Región del Maule - IX Región de la Araucanía - X Región de los Lagos, CODEPU, Santiago.

Schneider, Cathy (1991), "Mobilization at the grassroots: Shantytown and resistence in authoritarian Chile”, en Latin American Perspectives Vol. 18, No. 1, pp. 92-112. 
Ídem (1995), Shantytown Protest in Pinochet's Chile, Temple University Press, Philadelphia.

VVAA (2004), Humanidad y fe. Monseñor Carlos González Cruchaga. Homenaje en sus sesenta años de sacerdocio, Universidad de Talca, Talca.

Valdés, Teresa (1988), Venid, benditas de mi Padre. Las pobladoras, sus rutinas y sus sueños. FLACSO, Santiago.

Valdés, Teresa y Weinstein, Marisa (1993), Mujeres que Sueñan: las Organizaciones de Pobladoras en Chile, 1973-1989. FLACSO, Santiago.

Weinstein, Marisa (1996), Estado, Mujeres de Sectores Populares y Ciudadanía. FLACSO, Santiago.

Recibido: 24.01.2011

Aceptado: 03.03.2011 